\title{
Estudio Experimental y Computacional de la Cinética de Termólisis del 2,5-Hexanodiol
}

\author{
Adolfo E. Ensuncho, Nathaly Milanés y Juana R. Robles \\ Universidad de Córdoba, Departamento de Química, Cra 6 N76-103, Córdoba-Colombia. \\ (e-mail: aensuncho@sinu.unicordoba.edu.co; namios20@gmail.com; \\ juanarobles2003@yahoo.com)
}

Recibido Feb. 16, 2011; Aceptado Abr. 19, 2011; Versión Final recibida May. 03, 2011

\begin{abstract}
Resumen
La cinética de la reacción de descomposición térmica del 2,5-hexanodiol fue seguida por cromatografía de gases acoplada a espectrometría de masas bajo condiciones de pseudo-primer orden. El 2,5-hexanodiol fue sometido a termólisis a cinco temperaturas $(523,15,528,15,533,15$, 538,15 y $543,15 \mathrm{~K}$ ) y a su presión de vapor, obteniéndose como producto 2,5-dimetilfurano. La descomposición térmica resultó ser unimolecular, homogénea y de primer orden, con constantes de velocidad $(k)$ del orden de $10^{-4} \mathrm{~s}^{-1}$. El mecanismo de reacción propuesto fue corroborado por un estudio computacional a los niveles de teoría Hartree-Fock y funcionales de la densidad. El mecanismo de reacción propuesto transcurre a través de un estado de transición cíclico concertado. Las constantes de velocidad predichas con el nivel de teoría B3LYP/6-31G(d,p) estuvieron en buen acuerdo con valores experimentales.
\end{abstract}

Palabras clave: cinética, mecanismo de reacción, termólisis, química computacional, hexanodiol

\section{Experimental and Theoretical Study of the Thermolysis Kinetics of 2,5-Hexanediol}

\begin{abstract}
The kinetics of thermal decomposition reaction of 2,5-hexanediol was studied by gas chromatography coupled to mass spectrometry under conditions of pseudo-first order. The reactant 2,5-hexanediol was subjected to thermolysis at five temperatures $(523,15,528,15,533,15$, 538,15 y $543,15 \mathrm{~K}$ ) and at its vapor pressure, obtaining as product 2,5-dimethylfuran. The thermal decomposition was found to be unimolecular, homogeneous and of first order with rate constants $(k)$ of the order of $10^{-4} \mathrm{~s}^{-1}$. The proposed reaction mechanism was corroborated by a computational study at Hartree-Fock and Density Functional Theory levels. The mechanism proceeds through a concerted cyclic transition state. The rate constants predicted at the B3LYP/6-31G(d,p) level were in good agreement with the experimental values.
\end{abstract}

Keywords: kinetic, reaction mechanism, thermolysis, computational chemistry, hexanediol. 


\section{INTRODUCCIÓN}

El estudio de los compuestos polihidroxílicos tipo dioles ha generado un especial interés como materia prima en la industria de los polímeros por ser precursores de algunas fibras sintéticas utilizadas en la industria textil, cuya cadena base son algunos de estos alcoholes (Rogulska et al., 2007). Igualmente los dioles son utilizados en la industria biomédica como aditivos en la síntesis de biopolímeros (Pawlowski y Rokicki, 2004). El etilenglicol es el más elemental de esta serie de compuestos y presenta diversas aplicaciones, tales como solvente de pintura, refrigerante en la industria automotriz, entre otros (Verlag, 2000).

Para dioles alifáticos como el hexanodiol, se ha reportado el estudio del efecto de la temperatura sobre las propiedades volumétricas molares de exceso y volúmenes molares parciales de soluciones acuosas diluidas, mostrando con ello que los cambios en las posiciones de los grupos $\mathrm{OH}$ no causan cambios sensibles en dichas propiedades volumétricas (Romero et al., 2007). En el caso de algunos alcoholes polihidroxílicos, se ha estudiado la cinética de la pirólisis en fase gas, utilizando un sistema estático de alto vacio y se ha sugerido un mecanismo de reacción complejo (Chuchani et al., 1997). Sin embargo, a pesar de la importancia de estos dioles tanto a nivel industrial como biomédico, y al estudio de algunas propiedades en soluciones acuosas diluidas, se ha observado que estos compuestos han sido poco reportados en la literatura, por lo tanto, se requiere un mayor conocimiento sobre las estructuras moleculares que rigen el comportamiento reactivo de estos alcoholes. En razón a lo anterior, se realizó el estudio experimental y computacional de la cinética de termólisis del 2,5-hexanodiol a diferentes temperaturas.

\section{METODOLOGÍA}

El 2,5-hexanodiol, producto comercial Aldrich, fue secado y destilado bajo presión reducida, obteniéndose una pureza del $99 \%$ verificada con un equipo de cromatografía gaseosa acoplada a espectrometría de masas (GC-EM) Thermo Electron DSQII Single Quadrupole GC/MS, utilizándose una columna de $30 \mathrm{~m}$ de longitud, diámetro interno de $0.32 \mathrm{~mm}$, espesor de película de $0,25 \mu \mathrm{m}$ y límite de temperatura de $325^{\circ} \mathrm{C}$ específica para alcoholes. La termólisis del 2,5hexanodiol fue realizada en un minirreactor químico provisto de un horno cilíndrico acoplado a una termocupla con control electrónico de temperatura; el horno cilíndrico está recubierto de asbesto y material refractario con 18 perforaciones de un diámetro de $4 \mathrm{~mm}$ cada una, en el cual se introducen las ampollas con la sustancia a termolizar. Para la determinación de la cinética de reacción, se inyectaron aproximadamente $2,5 \mu \mathrm{L}$ de 2,5-hexanodiol en cada ampolla. Fueron selladas utilizando un soplete y cada cinética se conformó de 8 ampollas. Las ampollas se introdujeron en el sistema térmico a una temperatura previamente calibrada y retiradas a intervalos regulares de tiempo. Las muestras termolizadas se examinaron en el cromatógrafo gasmasas, usando como solvente 1,4-dioxano, debido a que en los cromatogramas no presentó superposición con los sustratos de estudio o con los productos formados por la reacción de descomposición térmica, los cuales se identificaron por espectrometría de masas.

La determinación de la constante de velocidad experimental se realizó mediante la ecuación (1) y corresponde a una reacción de primer orden.

$a-x=a e^{-k t}$

En donde a representa la concentración inicial y x la concentración al tiempo t.

Para la evaluación de las constantes de velocidad se utilizó la propiedad física $\lambda$. Incluyendo la propiedad física en la ecuación 1 se transforma en:

$\operatorname{Ln}=\left(\frac{\lambda_{\infty}-\lambda_{t}}{\lambda_{\infty}-\lambda_{0}}\right)=-k t$ 
Donde $\lambda$ es el área del pico correspondiente al 2,5-dimetilfurano para los tiempos (t) y tiempo infinito $\left(\mathrm{t}_{\infty}\right)$ respectivamente.

A partir de los valores de las constantes de velocidad, se realizó el gráfico de Arrhenius obteniéndose los valores de energía de activación (Ea) y factor frecuencia (A) por medio de la ecuación (3).

$$
k=A e^{-\frac{E a}{R T}}
$$

La ecuación (4) fue usada para calcular las constantes de velocidad para el equilibrio $\mathrm{k}^{\ddagger}$, así como la energía libre de Gibbs de activación $\Delta \mathrm{G}^{\ddagger}$ usando la ecuación (5). Los valores de entalpía y entropía de activación $\left(\Delta \mathrm{H}^{\ddagger}\right.$ y $\left.\Delta \mathrm{S}^{\ddagger}\right)$ se calcularon por medio de la ecuación (6).

$\mathrm{k}=\frac{\mathrm{k}_{\mathrm{B}} \mathrm{T}}{\mathrm{h}} \mathrm{k}^{\ddagger}$

$\Delta \mathrm{G}^{\ddagger}=-\mathrm{RTLnK}^{\ddagger}$

$\operatorname{Ln}\left(\frac{k}{T}\right)=\frac{\Delta S^{\ddagger}}{R}+\operatorname{Ln}\left(\frac{k}{h}\right)-\frac{\Delta H^{\ddagger}}{R}$

\section{Detalles computacionales}

Para corroborar las vías de reacción de la descomposición térmica del 2,5-hexanodiol se llevaron a cabo cálculos computacionales usando los métodos Hartree-Fock (Cavaliere y De Giovannini, 2010) y el funcional hibrido de correlación e intercambio B3LYP (Koch, 2001) implementados en el paquete Gaussian03 (Frisch, 2004). Las optimizaciones de las geometrías y cálculos de frecuencia, se realizaron con un conjunto de funciones base doble $\zeta$ de valencia dividida 6$31 \mathrm{G}(\mathrm{d}, \mathrm{p})$ (Klein y Zottola, 2006), incluyendo polarización en átomos pesados y ligeros. Todos los puntos estacionarios localizados sobre la superficie de energía potencial se caracterizaron como mínimos o estados de transición de primer orden (caracterizado por tener solo una frecuencia imaginaria). La vía de reacción fue seguida por el método de la coordenada intrínseca de reacción (Fukui, 1981) en pasos de $0,1 \mathrm{amu}^{1 / 2}$ bohr de la geometría del estado de transición conectando dos mínimos. Las constantes de velocidad se calcularon mediante la teoría del estado de transición, de acuerdo con el formalismo propuesto por Eyring (Connors, 1990) (ecuación 7) donde $\mathrm{k}_{\mathrm{B}}$ es la constante de Boltzmann, $T$ es la temperatura absoluta, $\mathrm{h}$ la constante de Planck y $\Delta \mathrm{G}^{\ddagger}$ es la energía libre de activación de Gibbs para la reacción de descomposición térmica.

$k=\frac{k_{B} T}{h} e^{-\frac{\Delta G^{\ddagger}}{R T}}$

\section{RESULTADOS Y DISCUSIÓN}

La reacción de descomposición térmica del 2,5-hexanodiol se muestra en la figura 1.

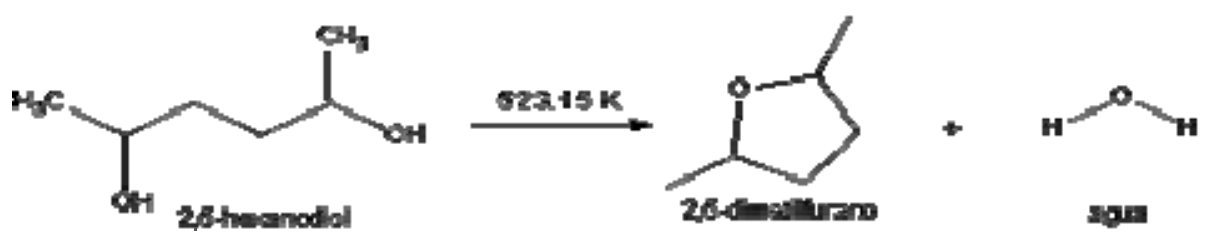

Fig. 1: Termólisis de 2,5-hexanodiol 
Las gráficas de $\operatorname{Ln}\left(\lambda_{\infty}-\lambda_{t}\right)$ vs t, realizadas a diferentes temperaturas (figura 2), mostraron una buena correlación lineal confirmando que la reacción de descomposición térmica está de acuerdo con una ecuación cinética de primer orden.

Los valores de las constantes de velocidad $k_{o b s}$ presentaron proporcionalidad con la temperatura, puesto que al incrementar la temperatura la velocidad de la reacción aumentaba. Los valores de las constantes de velocidad para cada temperatura se presentan en la tabla 1. Estas constantes de velocidad experimentales fueron comparadas con las constantes de velocidad predichas al nivel de teoría B3LYP/6-31G(d,p), mostrando un buen acuerdo, siendo por tanto este nivel de teoría el que mejor describe el sistema estudiado.
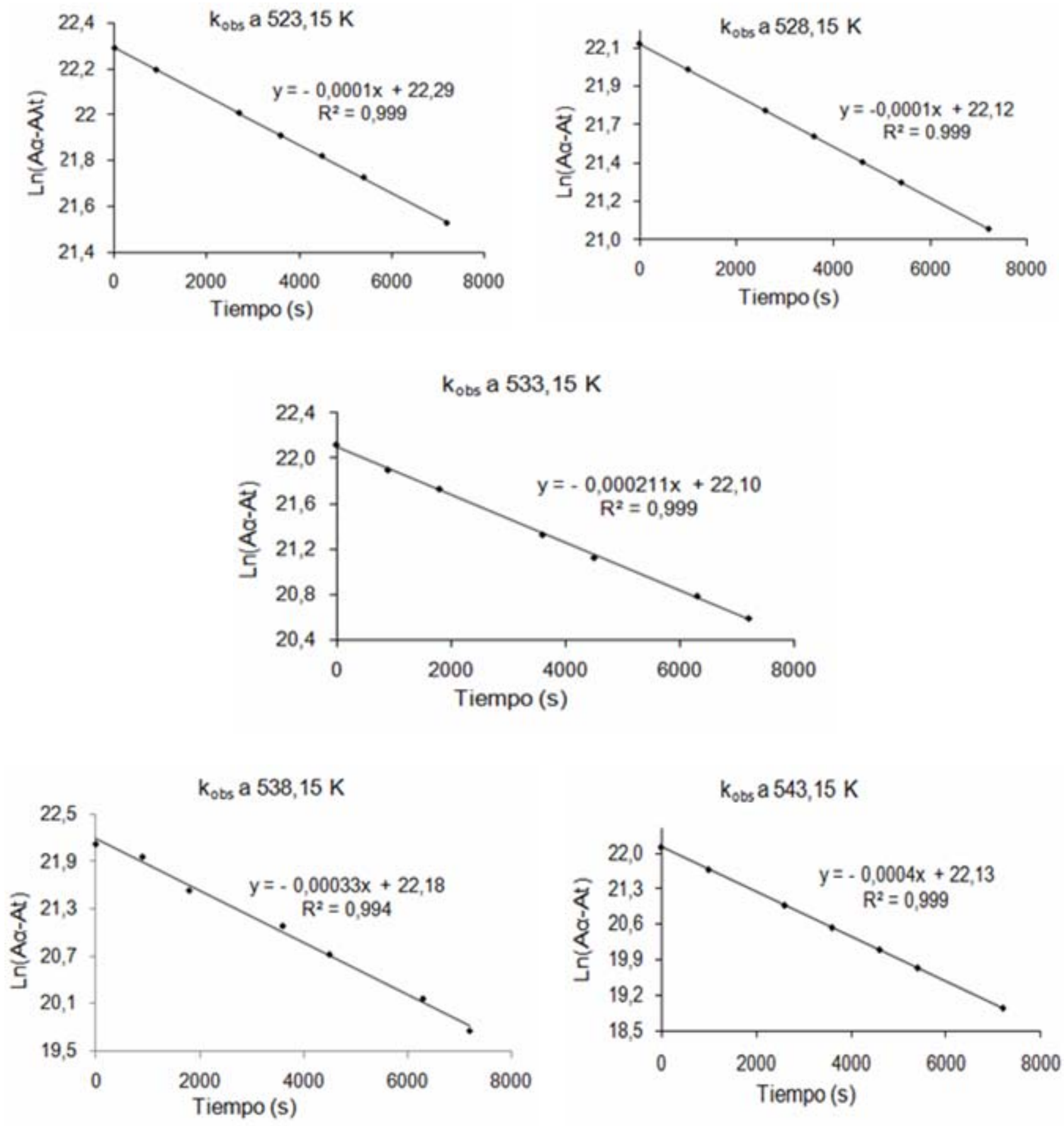

Fig. 2: $\mathrm{K}_{\mathrm{obs}}$ para la termólisis de 2,5-hexandiol a diferentes Temperaturas 
Tabla 1: Constantes de velocidad para la termólisis del 2,5-hexanodiol obtenidas experimentalmente y predichas a los niveles de teoría HF/6-31G(d,p) y B3LYP/6-31G(d,p).

\begin{tabular}{|c|c|c|c|}
\hline \multirow{3}{*}{$\mathrm{T}(\mathrm{K})$} & \multicolumn{3}{|c|}{$k\left(s^{-1}\right)$} \\
\hline & \multicolumn{2}{|c|}{ Computacional } & \multirow{2}{*}{ Experimental } \\
\hline & $\mathrm{HF}$ & DFT & \\
\hline 523.15 & $3.3 \times 10^{-2}$ & $2.875 \times 10^{-4}$ & $(1.06 \pm 0.11) \times 10^{-4}$ \\
\hline 528.15 & $4.5 \times 10^{-2}$ & $4.043 \times 10^{-4}$ & $(1.47 \pm 0.09) \times 10^{-4}$ \\
\hline 533.15 & $6.0 \times 10^{-2}$ & $5.651 \times 10^{-4}$ & $(2.11 \pm 0.12) \times 10^{-4}$ \\
\hline 538.15 & $8.2 \times 10^{-2}$ & $8.240 \times 10^{-4}$ & $(3.13 \pm 0.09) \times 10^{-4}$ \\
\hline 543.15 & $1.04 \times 10^{-1}$ & $1.083 \times 10^{-3}$ & $(4.42 \pm 0.01) \times 10^{-4}$ \\
\hline
\end{tabular}

Tabla 2: Valores de $\mathrm{Ln} \mathrm{k} / \mathrm{s}^{-1}$ y $1 / \mathrm{T} \times 10^{-3} / \mathrm{K}^{-1}$.

\begin{tabular}{cccc}
\hline $\mathrm{T}(\mathrm{K})$ & $\mathrm{k}\left(\mathrm{s}^{-1}\right)$ & $\mathrm{Ln} \mathrm{k} / \mathrm{s}^{-1}$ & $1 / \mathrm{T} \times 10^{-3} / \mathrm{K}^{-1}$ \\
\hline 523.15 & $1.06 \times 10^{-4}$ & -9.152 & 1.911 \\
528.15 & $1.47 \times 10^{-4}$ & -8.825 & 1.893 \\
533.15 & $2.11 \times 10^{-4}$ & -8.464 & 1.876 \\
538.15 & $3.13 \times 10^{-4}$ & -8.069 & 1.858 \\
543.15 & $4.42 \times 10^{-4}$ & -7.724 & 1.841 \\
\hline
\end{tabular}

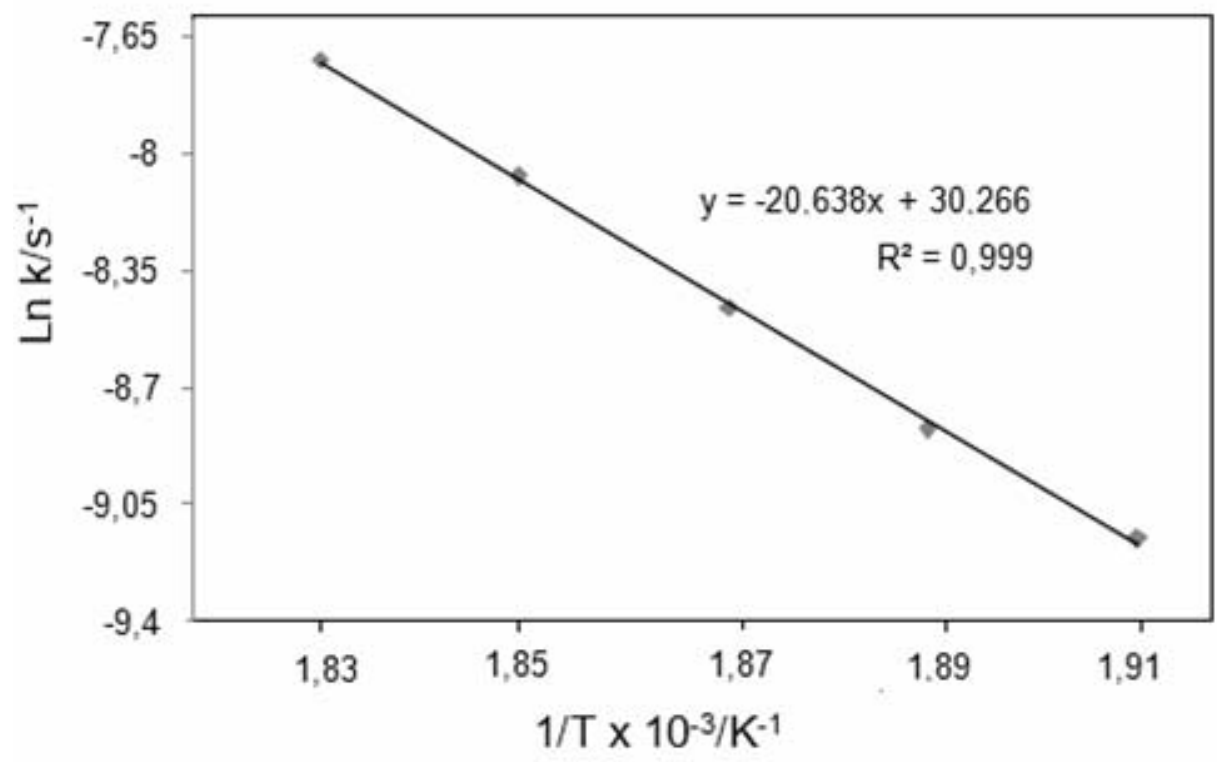

Fig. 3: Gráfico de Arrhenius

En la tabla 2 y figura 4, se muestra la gráfica de Arrhenius las cuales relacionan los valores de las constantes de velocidad con respecto a las temperaturas de trabajo, obteniéndose una línea recta con pendiente igual a -Ea (energía de activación) y un intercepto correspondiente al factor frecuencia (A). La gráfica presentó buena correlación para la descomposición térmica del 2,5- 
hexanodiol. Los parámetros termodinámicos fueron calculados mediante la ecuación de Eyring. Así, en la tabla 3 , se presentan los parámetros termodinámicos calculados a la temperatura de $543,15 \mathrm{~K}$ para el 2,5-hexanodiol. El valor negativo de la entropía de activación, indica que el estado de transición es más ordenado que los reactivos y los valores positivos para la entalpía de activación y energía de activación indican que la reacción de descomposición es endergónica, estable, no espontánea y con energía interna no suficiente para que la reacción química se lleve a cabo a condiciones normales. El valor de energía de activación Ea es cercano al cambio de entalpía de activación, de acuerdo con la expresión Ea $=\Delta \mathrm{H}^{\ddagger}+\mathrm{nRT}$. El valor del factor preexponencial $A$, se encontró en el intervalo $10^{11}-10^{13} \mathrm{~s}^{-1}$, lo cual es característico para reacciones de primer orden (Julio et al., 2007).

Tabla 3: Parámetros termodinámicos del 2,5-hexanodiol a 543,15 K.

\begin{tabular}{|c|c|c|c|c|c|}
\hline $\begin{array}{c}\text { Parámetro } \\
\text { Termodinámico }\end{array}$ & $\begin{array}{c}\mathrm{Ea} \\
\left(\mathrm{kJ} \mathrm{mol}^{-1}\right)\end{array}$ & $\begin{array}{c}\mathrm{A} \\
\left(\mathrm{s}^{-1}\right)\end{array}$ & $\begin{array}{c}\Delta \mathrm{G}^{\ddagger} \\
\left(\mathrm{kJ} \mathrm{mol}^{-1}\right)\end{array}$ & $\begin{array}{c}\Delta \mathrm{H}^{\ddagger} \\
\left(\mathrm{kJ} \mathrm{mol}^{-1}\right)\end{array}$ & $\begin{array}{c}\Delta \mathrm{S}^{\ddagger} \\
\left(\mathrm{kJ} \mathrm{K}^{-1} \mathrm{~mol}^{-1}\right)\end{array}$ \\
\hline Valor & 171,417 & $1,271 \times 10^{-3}$ & 170,813 & 166,994 & $-7,178 \times 10^{-3}$ \\
\hline
\end{tabular}

De acuerdo con los valores obtenidos de los parámetros termodinámicos y los productos de la reacción de descomposición térmica del 2,5-hexanodiol, se propone un mecanismo de reacción que involucra un estado de transición cíclico concertado, en una etapa, formando el 2,5dimetilfurano, como el producto principal y una molécula de agua, como se muestra en la figura 4.

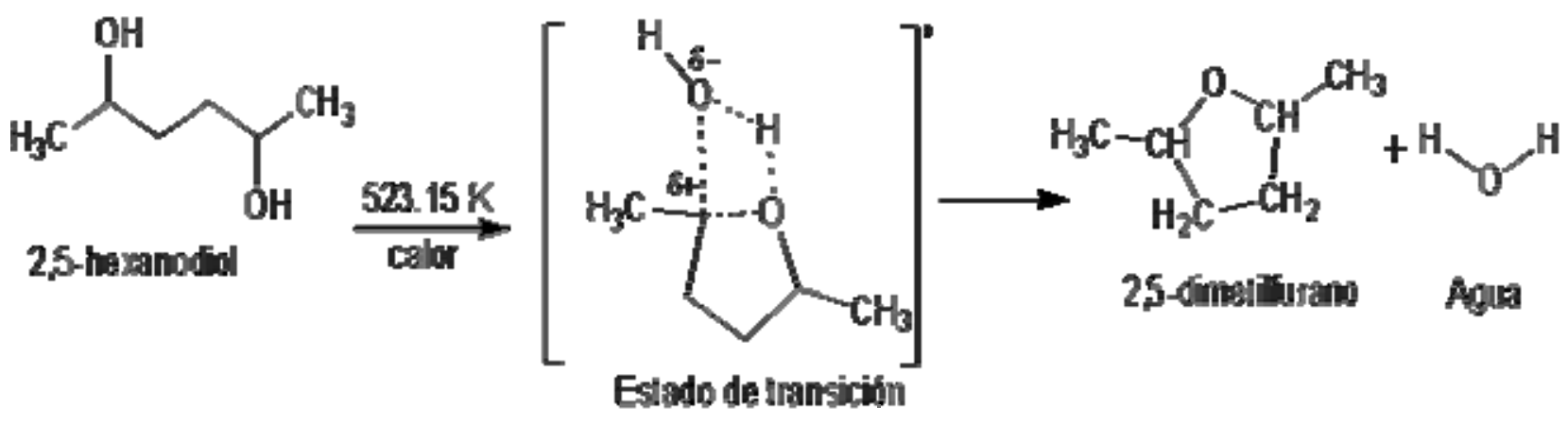

Fig. 4: Mecanismo propuesto para la reacción de termólisis del 2,5-hexanodiol

Para respaldar el mecanismo propuesto con base a la información experimental (tablas 2 y 3 ), se llevaron a cabo cálculos mecano-cuánticos para la descomposición térmica del 2,5-hexanodiol; usando Hartree-Fock y DFT. Se realizaron cálculos para obtener los perfiles de reacción determinados experimentalmente. Cada estructura estacionaria sobre la superficie de energía potencial correspondiente a mínimos locales de energía y estados de transición de primer orden se caracterizaron mediante cálculos de frecuencia. El estado de transición de la termólisis del 2,5hexanodiol, se caracterizó por tener solo una frecuencia imaginaria de $632,9 \mathrm{i} \mathrm{cm}^{-1}$, lo cual hace referencia a que un grado de libertad vibracional de los $3 \mathrm{~N}-6$ grados en el estado de transición, se transforma en un grado de libertad traslacional -modo imaginario- conduciéndolo posteriormente a la formación de los productos (Ball, 2003). Asimismo, se determinó la conectividad de las diferentes etapas para la reacción de termólisis del 2,5-hexanodiol, por análisis de la coordenada intrínseca de reacción (Fukui, 1981) -IRC-, utilizando la teoría del funcional de la densidad -DFT-, como se muestra en la figura 5. Esto se hizo con el fin de corroborar que el estado de transición localizado, conectaba con los correspondientes puntos estacionarios asociados al reactante y los productos. 


\section{Coordenada de Reacción}

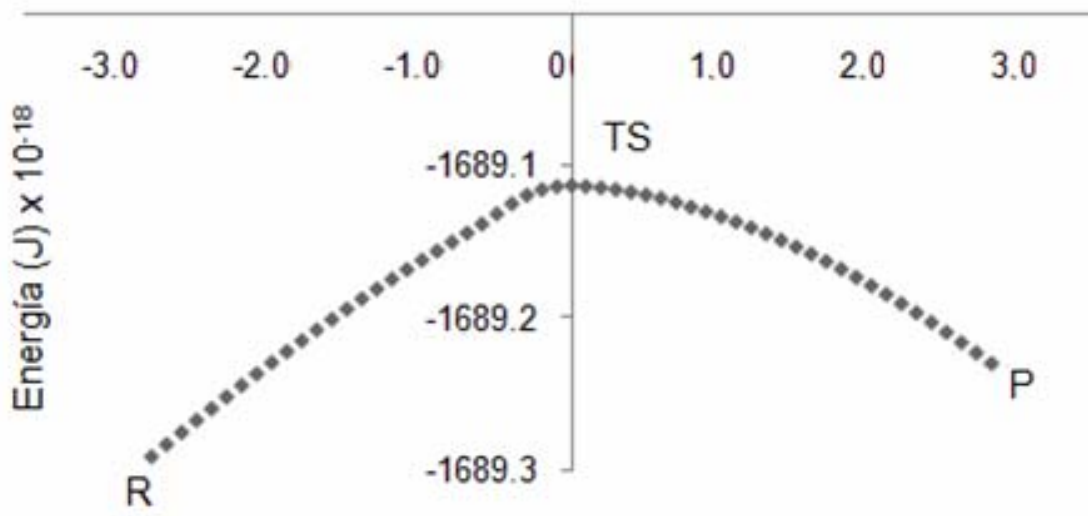

Fig. 5: Análisis IRC para la termólisis del 2,5-hexanodiol, calculado a B3LYP/6$31 \mathrm{G}(\mathrm{d}, \mathrm{p}) \mathrm{R}=$ Reactivo, $\mathrm{TS}=$ Estado de transición, $\mathrm{P}=$ Producto

\section{CONCLUSIONES}

Los parámetros termodinámicos determinados en este estudio son consistentes con el mecanismo propuesto para el 2,5-hexanodiol, en donde se puede decir que es un proceso endergónico, en una etapa que es la determinante de la velocidad de reacción, con alta energía de activación; la entropía obtenida experimentalmente en la termólisis del 2,5-hexanodiol presenta un valor negativo, indicando que esta reacción se lleva a cabo por medio de un estado de transición cíclico, más ordenado que su estado inicial, con posterior reordenamiento del mismo.

Se puede concluir que la velocidad de la descomposición térmica del 2,5-hexanodiol, aumenta con el incremento de la temperatura, lo cual es consistente con la ecuación de Arrhenius. Al comparar los métodos experimentales y teóricos usados en este trabajo, se observa que los datos de los parámetros termodinámicos obtenidos experimentalmente se comparan muy bien con los calculados al nivel B3LYP/6-31G(d,p), lo que corrobora los resultados experimentales, confirmando la complementariedad de los métodos utilizados.

\section{AGRADECIMIENTOS}

Centro de investigación de la Universidad de Córdoba (CIUC), por la financiación de la investigación. A los doctores del Instituto de Investigaciones Científicas (IVIC) Gabriel Chuchani y José R. Mora por la cuidadosa lectura de este manuscrito.

\section{REFERENCIAS}

Ball, D., Physical chemistry, Thomson-Brooks/Cole, Pacific Grove CA (2003).

Cavaliere, F. y U. De Giovannini., General Hartree-Fock method and symmetry breaking in quantum dots, Physica E 42, 606-609 (2010).

Chuchani, G., R.M. Dominguez., A. Rotinov y I. Martin., The gas-phase pyrolysis of several aliphatic 1,3-diols, The kinetic and mechanism of 2,4-dimethyl-2,4-pentanediol, Int. J. Chem. Kinet, 29, 851-854 (1997).

Connors, K., Chemical kinetics the study of reaction rates in solution, VCH Publ, New York (1990).

Fukui, K., The path of chemical reactions the IRC approach, Accounts of Chemical Research 14, 363-368 (1981). 
Frisch, M., G.W., Trucks y otros 80 autores; Gaussian03, Revision E.01, Gaussian, Inc, Wallingford CT, Pittsburgh, USA (2004).

Julio, L.L., A. Herize., M. Tosta., R.M. Domínguez., J. Lafont y G. Chuchani., The unimolecular elimination kinetics of benzaldoxime in the gas phase, Int. J. Chem. Kinet. 39, 145-147 (2007).

Klein, R.A. y M.A. Zottola., Pople versus Dunning basis-sets for group IA metal hydrides and some other second row hydrides: The case against a De Facto standard, Chem. Phys. Lett., 419, 254258 (2006).

Koch, W., A chemist's guide to density functional theory, Wiley-VCH, Weinheim, New York (2001).

Pawlowski, P. y G. Rokicki., Synthesis of oligocarbonate diols from ethylene carbonate and aliphatic diols catalyzed by alkali metal salts, Polymer 45, 3125-3137 (2004).

Rogulska, M., A. Kultys y W. Podkoscielny., Studies on thermoplastic polyurethanes based on new diphenylethane-derivative diols. II. Synthesis and characterization of segmented polyurethanes from HDI and MDI, Eur. Polym. J., 43, 1402-1414 (2007).

Romero, C.M., M.S. Páez., J.C. Arteaga., M.A. Romero y F. Negrete., Effect of temperature on the volumetric properties of dilute aqueous solutions of 1,2-hexanediol, 1,5-hexanediol, 1,6hexanediol, and 2,5-hexanediol, J. Chem. Thermodyn., 39, 1101-1109 (2007).

Verlag, G., Ullmann's Encyclopedia of Industrial Chemistry, $6^{\text {a }}$ edición, Wiley-VCH, Weinheim, Germany (2000). 